\title{
FRACTAL DIMENSION ESTIMATION FROM FULLY POLARIMETRIC SAR DATA
}

\author{
Gerardo Di Martino $^{1}$, Giorgio Franceschetti ${ }^{l}$, Antonio Iodice ${ }^{1}$, \\ Daniele Riccio ${ }^{1}$, Giuseppe Ruello ${ }^{1}$, Ivana Zinno ${ }^{2}$ \\ ${ }^{1}$ Università di Napoli Federico II - DIETI, Napoli, Italy \\ ${ }^{2}$ Istituto per il Rilevamento Elettromagnetico dell'Ambiente (IREA), CNR, Napoli, Italy
}

\begin{abstract}
Fractal dimension is widely recognized as key parameter for the geophysical characterization of natural surfaces. A technique for estimating the fractal dimension of a surface from range cuts of an amplitude SAR image has been recently developed by the authors. In this paper, we explore the potentiality of using fully polarimetric SAR data for the retrieval of the fractal dimension along the azimuth direction. This is obtained exploiting the Polarimetric TwoScale model for the description of polarimetric data. In particular, specific polarimetric combinations are considered, which provide images with strong dependence on the azimuth slopes of the observed surface. The proposed technique is tested on actual fully polarimetric AirSAR Lband data.
\end{abstract}

Index Terms - SAR, polarimetry, fractals

\section{INTRODUCTION}

In this paper, we explore the potentiality of using fully polarimetric SAR data for the estimation of the fractal dimension [1] of an observed surface. In previous works [2][3], the authors introduced new models to describe the imaging of natural surfaces, allowing the estimation of the fractal dimension directly from a SAR amplitude image. This estimation is performed exploiting a sliding window scouring the whole image, thus allowing the generation, as output, of a new value-added SAR product, the fractal dimension map, i.e., a point-by-point map of the estimated fractal dimension of the imaged surface [3]. The model is based on a (band-limited) fractional Brownian motion (fBm) description of the observed surface and on the observation that, due to the side-looking geometry of the sensor, the radar returns and, hence, the amplitude SAR images depend only on the range slopes of the observed surface, at least as a first order approximation. Therefore, within this approximation, the effect of azimuthal slopes can be neglected and the algorithm performs a spectral analysis based only on range cuts of the SAR amplitude image. Since the $\mathrm{fBm}$ has a power-law spectrum, also its (properly defined) derivative has a power-law spectrum and the spectral exponent is related to the fractal dimension [3].
Accordingly, the estimation of the fractal dimension of the surface can be obtained through a linear regression of the spectrum in a log-log plane [3].

In the last decades, many studies demonstrated the effectiveness of SAR polarimetry for the investigation of key physical properties of an observed surface. For this reason, all the major ongoing and future spaceborne SAR missions are projected to include some kind of polarimetric capabilities, and, in many cases, fully polarimetric acquisition capabilities are enforced. In this work, we are mainly interested in fully polarimetric acquisitions, due to the crucial role of surface azimuth slopes for fully polarimetric applications [4]-[5]. In particular, azimuth slope variations are the main responsible for polarization orientation shifts [4], which consist in rotations of the polarization plane around the sensor line of sight. In fact, the sensitiveness of these data to the azimuth slopes also led to the development of techniques aimed at estimating azimuth height profiles from fully polarimetric SAR data [5]. This dependence can also be exploited for fractal dimension estimation, provided that an adequate scattering model is considered: the model must take into account the presence of rotations of the polarization plane [6]-[7].

In this paper, we use the Polarimetric Two-Scale Model (PTSM) proposed in [6] to describe the behavior of polarimetric SAR data. In particular, we show how it is possible to obtain from these data a map of the fractal dimension estimated along the azimuth direction, which can be potentially used in conjunction to the standard one estimated exploiting the range cuts of the original image. The considered theoretical model is briefly introduced in the next section, while in Section 3 we show the first results regarding the application of the proposed algorithm to AirSAR L-band fully polarimetric data.

\section{POLARIMETRIC MODEL}

The first-order small perturbation method (SPM) is a theoretical scattering model adequate for SAR electromagnetic modeling [8], whose range of applicability has been also adapted to fractal surfaces [9]. However, the first-order SPM fails to describe cross-polarization and depolarization effects present in actual data [6]-[7]. To take into account these phenomena we consider the PTSM 
model, which extends the first-order SPM, taking into account the presence of a non-null angle of rotation of the local incidence plane around the sensor line of sight, $\beta$ [6]. This rotation angle, in a first order approximation, depends on the azimuth slopes of the observed surface [4], [6]. This kind of dependence can be exploited for fractal dimension maps synthesis. In fact, when fully polarimetric SAR data are available, we can synthesize the following image as a combination of elements of the scattering matrix $S$ :

$$
I_{1}=\frac{2 S_{H V}}{S_{V V}-S_{H H}} .
$$

Considering the expression of the scattering matrix elements according to the PTSM model [6], we obtain that

$$
I_{1}=\frac{2 \operatorname{tg} \beta}{1-\operatorname{tg}^{2} \beta}=\operatorname{tg} 2 \beta \text {, }
$$

where, moreover, the angle $\beta$ is related to the azimuth slope $q$, range slope $p$, and sensor look angle $\vartheta_{0}$ by the following expression:

$$
\operatorname{tg}(\beta)=\frac{q}{-p \cos \vartheta_{0}+\sin \vartheta_{0}}
$$

Using expression (3) in conjunction with (2), it is easy to demonstrate that in a first order approximation $I_{1}$ depends only on the azimuth slopes of the observed surface. Therefore, $I_{1}$ can be exploited for the estimation of the fractal dimension of the surface, using the same algorithm proposed in [3], provided that the spectral analysis is performed on azimuth cuts of the image, rather than on range cuts. The possibility to estimate the fractal dimension of the surface in the azimuth direction can be used to evaluate the presence of anisotropy in the surface roughness and/or to increase the accuracy of the range estimated fractal dimension.

However, use of (1) can be problematic due to the noisy character of the single-look HV channel, and to the potential presence of singularities when the difference between $\mathrm{VV}$ and $\mathrm{HH}$ channels is almost null [4]. For this reason, in many practical situations better results can be obtained using a combination of elements of the polarimetric coherency matrix $\boldsymbol{T}[4]$ :

$$
I_{2}=\frac{4 \operatorname{Re}\left(\left\langle\left(S_{H H}-S_{V V}\right) S_{H V}^{*}\right\rangle\right)}{4\left\langle\left|S_{H V}\right|^{2}\right\rangle-\left\langle\left|S_{H H}-S_{V V}\right|^{2}\right\rangle},
$$

where $\langle\cdot\rangle$ stands for statistical mean, $\operatorname{Re}(\cdot)$ for real part, and use is made of two diagonal terms of $\boldsymbol{T}$ (denominator) and of the real part of an off-diagonal term of $\boldsymbol{T}$ (numerator) [6]. Note that if only multi look complex (MLC) data are available, the image in (1) cannot be generated, while (4) can be easily obtained starting from the elements of $\boldsymbol{T}$. It is easy to demonstrate that starting from (4) an expression similar to the one in (2) is obtained: in particular,

$$
I_{2}=\operatorname{tg} 4 \beta
$$

and, also in this case, using (3), a first-order dependence from the azimuth slope only is present. The image $I_{2}$ can be processed with the algorithm proposed in [3], provided that the spectral analysis is performed on azimuth cuts of the image, rather than on range cuts. Note that, as in the previous case, problems can be present when the difference between VV and $\mathrm{HH}$ channels is almost null: this happens especially in areas of high positive range slopes, where, in presence of an almost specular scattering scenario, $S_{V V} \simeq$ $S_{H H}$, and the sensitiveness of (4) to azimuth slopes is very low. Actually, in these areas the speckle level is higher (because the radar return is higher), and we face both low sensitiveness to azimuth slopes and high speckle noise levels [4]. Moreover, (5) involves the evaluation of the coherency matrix, i.e. spatial averaging is applied to the data. Therefore, estimation of the fractal dimension using $I_{2}$ could be affected by some degree of distortion of the fractal characteristics of the surface due to the presence of multilook.

\section{EXPERIMENTAL RESULTS}

We present here the first results obtained from the analysis of one AirSAR L-band fully polarimetric SAR image acquired in the area of Camp Roberts, California (USA). The data were available only in MLC format, so that only the elements of the coherency matrix are available. In Fig. 1 (a) we show the $\mathrm{HH}$ amplitude channel of the image, while in Fig. 1 (b) the polarimetric composition image $I_{2}$ of (4) is reported. It is evident that the image in Fig. 1 (b) presents high levels of noise in areas with strong positive range slopes, as also discussed in the previous section.

First, we estimated the fractal dimension map from the amplitude image of the $\mathrm{HH}$ channel with the standard technique of [3]. The obtained result is reported in Fig. 2 (a). We then tested the approach proposed in the previous section. In this case, the use of the image combination $I_{1}$ is not possible with the available MLC data, which allow only the synthesis of the polarimetric composition $I_{2}$, reported in Fig. 1 (b). From this image we then estimated the fractal dimension map according to the rationale discussed in the previous section. In particular, the estimation was performed on $\operatorname{tg}\left(\operatorname{tg}^{-1}\left(I_{2}\right) / 4\right)$. The resulting map is reported in Fig. 2 (b). From the visual inspection of Fig. 2 it can be noted that a certain degree of correlation is present between the two maps, even if the overall appearance is quite different. This difference is probably due to the different effect of geometrical distortions (i.e., layover, foreshortening, and shadowing) on cuts in azimuth with respect to those in range. In addition, the map obtained from the $\mathrm{HH}$ image is significantly affected by the presence of strong scatterers, that appear as bright points in this image, whereas in the polarimetric composition $I_{2}$ they are almost completely singled out due to the ratio appearing in (4). The overall 
effect is that in the map of Fig. 2 (a) the degree of correlation between adjacent pixels is stronger.

In Fig. 3 the histograms of the two estimated maps are shown. The mean values of the two maps are 2.14 for the $\mathrm{HH}$-polarization image (estimated using range cuts) and 2.30 for the $I_{2}$ image (estimated using azimuth cuts). This difference can be explained partly by the noisier character of the map in Fig. 2 (b) (in fact, noise tends to increase the estimated value of $D[3]$ ) and in part by the different impact of geometric distortions on the evaluation of the two maps, as discussed before. Similar considerations hold for the maps' standard deviations, which are equal to 0.13 for the map in Fig. 2 (a) and 0.07 for the one in Fig. 2 (b): again, this difference is probably mainly due to the different impact of geometrical distortions due to the slant range acquisition geometry of the system during the spectral estimation step. At the moment, no definitive conclusion about potential anisotropies of the fractal behavior of the surface can be drawn.

In conclusion, the results of the estimation strongly depend on the quality of the image $I_{2}$ used for the estimation in terms of signal-to-noise ratio. Better results could be obtained using P-band data (which at this time are not yet available to the authors): in fact, the possibility to obtain good estimates of the polarimetric orientation angle is also hampered by the potential presence of vegetation [4], whose scattering is weaker at lower frequency.

\section{CONCLUSIONS}

In this paper, we investigated the possibility of obtaining maps of the fractal dimension of a natural surface estimating it from cuts along the azimuth direction of a polarimetricbased SAR image composition. These new maps could be potentially used in conjunction to the standard ones, estimated exploiting the range cuts of the original image. The presented results, although preliminary, demonstrate the potentialities of the proposed approach for fractal dimension estimation from fully polarimetric SAR data. In perspective, the fractal dimension maps estimated along the azimuth direction can provide an indication of anisotropy of the fractal characteristics of an observed surface. However, the impact of both speckle and thermal noise and the different role of geometric distortions within the spectral estimation step are two issues that need to be further investigated.

\section{REFERENCES}

[1] B. B. Mandelbrot, The Fractal Geometry of Nature. New York: Freeman, 1983.

[2] G. Di Martino, A. Iodice, D. Riccio, and G. Ruello, "Imaging of Fractal Profiles", IEEE Trans. Geosci. Remote Sens., vol. 48, no. 8, pp. 3280-3289, Aug. 2010.

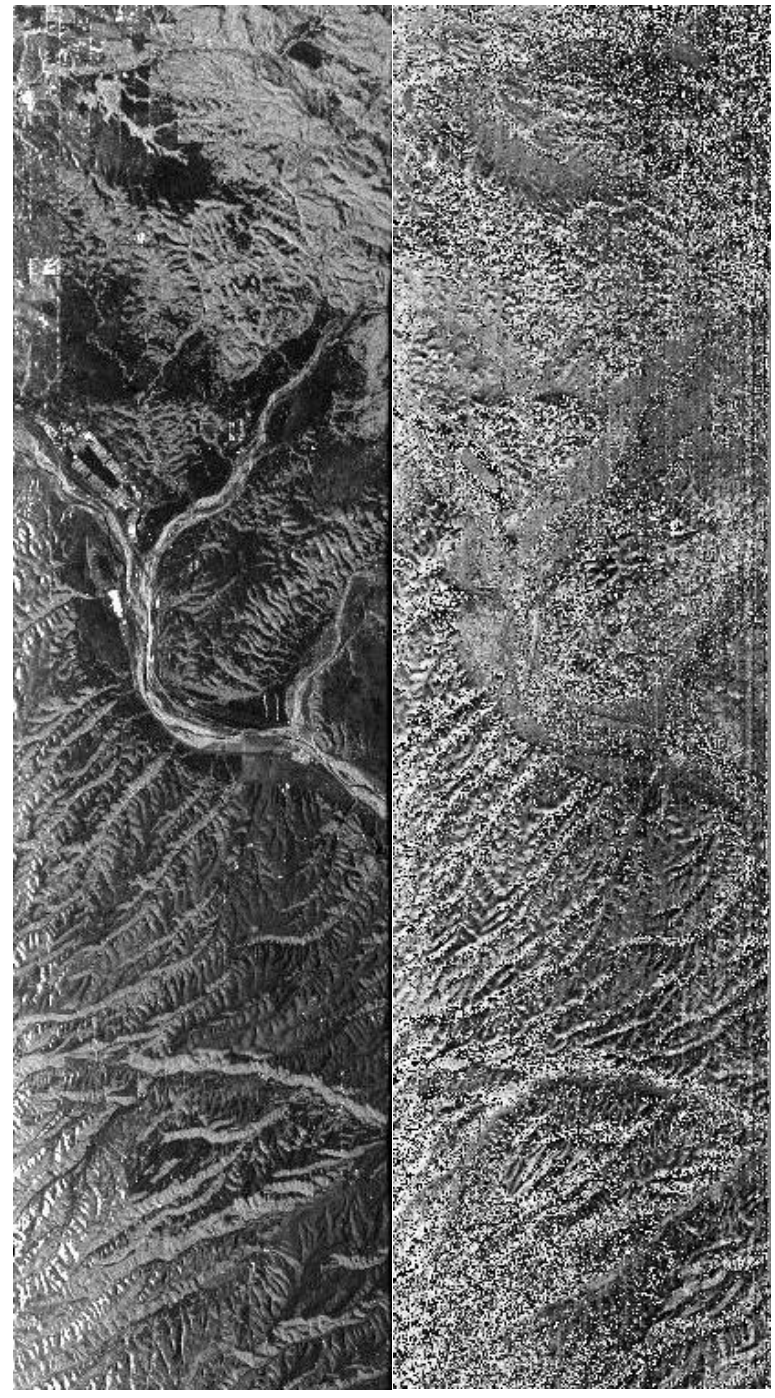

(a)

(b)

Fig. 1 Subset of the HH-polarization amplitude image (a) and the associated $I_{2}$ image (b). A $5 \times 5$ spatial multilook is applied.

[3] G. Di Martino, D. Riccio, and I. Zinno, "SAR Imaging of Fractal Surfaces", IEEE Trans. Geosci. Remote Sens., vol. 50, no. 2, pp. 630-644, Feb. 2012.

[4] D. L. Schuler, T. L. Ainsworth, E. Krogager, D. Kasilingam, and W.-M. Boerner, "On the estimation of radar polarization orientation shifts induced by terrain slopes," IEEE Trans. Geosci. Remote Sens., vol. 40, no. 1, pp. 30-41, Jan. 2002.

[5] D. L. Schuler, J. Lee, and G. De Grandi, "Measurement of Topography Using Polarimetric SAR Images," IEEE Trans. Geosci. Remote Sens., vol. 34, no. 5, pp. 1266-1277, Sep. 1996.

[6] A. Iodice, A. Natale, and D. Riccio, "Retrieval of Soil Surface Parameters via a Polarimetric Two-Scale Model," IEEE Trans. Geosci. Remote Sens., vol. 49, no. 7, pp. 2531-2547, July 2011. 
[7] I. Hajnsek, E. Pottier, and S. R. Cloude, "Inversion of surface parameters from polarimetric SAR," IEEE Trans. Geosci. Remote Sens., vol. 41, no. 4, pp. 727-744, Apr. 2003.

[8] F. T. Ulaby, R. K. Moore, and A. K. Fung, Microwave Remote Sensing: Active and Passive. Reading, MA: Addison-Wesley, 1982.

[9] G. Franceschetti and D. Riccio, Scattering, Natural Surfaces and Fractals. Burlington, MA: Academic Press, 2007.

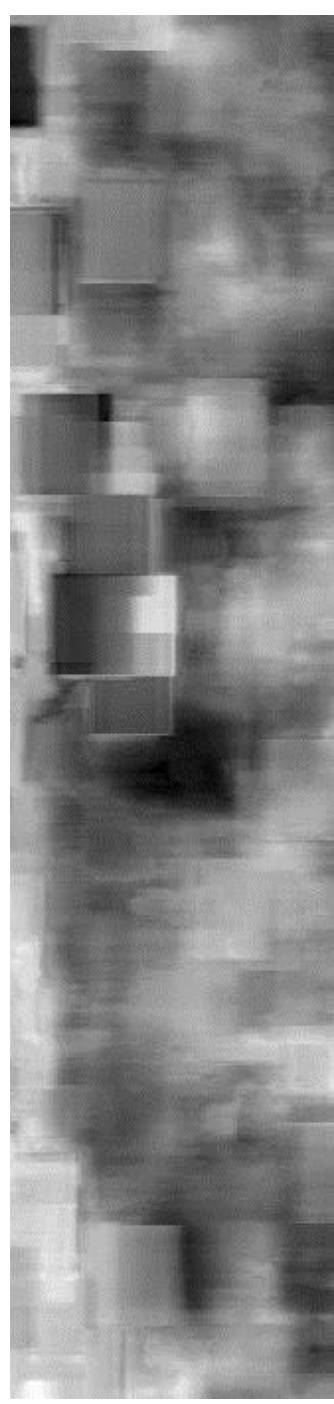

(a)

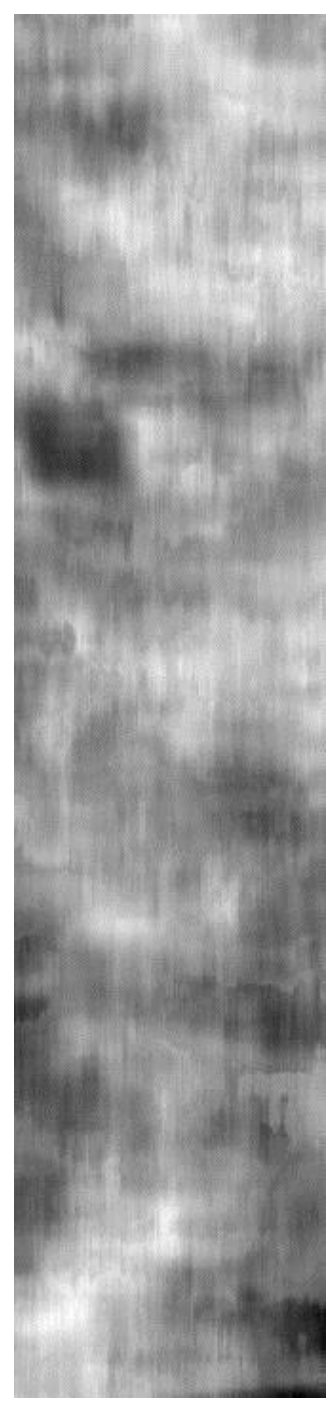

(b)
Fig. 2 Fractal dimension maps obtained from the considered subset of the HH-polarization amplitude image with standard technique (a) and from $I_{2}$ with the proposed method (b).

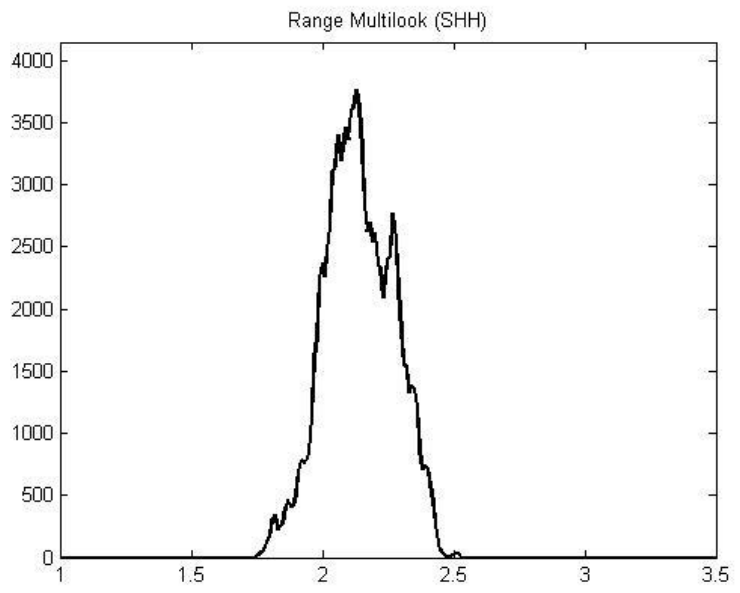

(a)

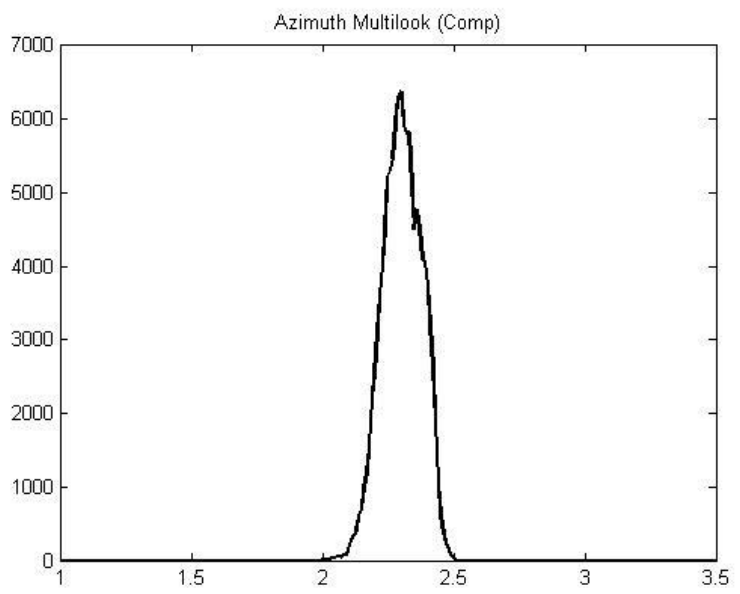

(b)

Fig. 3 Histograms of the fractal dimension maps reported in Fig. 2: $\mathrm{HH}$ amplitude image (a) and $I_{2}$ image (b). 